\title{
Polymer conformation in supramolecular complexes with wedge-shaped ligands: exploring the impact of the liquid-crystalline organization
}

Denis V. Anokhin, $* \dagger+\ddagger \S$ Kseniia N. Grafskaia, ${ }^{\S}$ Inessa A. Izdelieva, ${ }^{\&}$ Xiaomin Zhu, ${ }^{\nabla}$ Dimitri A. Ivanov $\uparrow, \star,, \|$

${ }^{\dagger}$ Lomonosov Moscow State University, Faculty of Fundamental Physical and Chemical Engineering, GSP-1, Leninskie Gory 1, Moscow, 119991, Russia

$\sharp$ Institute of Problems of Chemical Physics, Russian Academy of Sciences, Semenov Av. 1, Chernogolovka, 142432, Russia

$\S$ Moscow Institute of Physics and Technology, Institutskiy per. 9, Dolgoprudny, 141700, Russia

${ }^{\&}$ Lomonosov Moscow State University, Faculty of Chemistry, GSP-1, 1-3 Leninskie Gory 1, Moscow, 119991, Russia

${ }^{\nabla}$ DWI-Leibniz-Institute for Interactive Materials e.V. and Institute for Technical and Macromolecular Chemistry of RWTH Aachen University, Forkenbeckstr. 50, D-52056 Aachen, Germany

"Institut de Sciences des Matériaux de Mulhouse-IS2M, CNRS UMR 7361, 15 Jean Starcky, 68057 Mulhouse, France

*Email:deniano@yahoo.com

\begin{abstract}
:
The chain conformation of poly(2-vinylpyridine) (P2VP) complexed by 4-((4-((3,4,5tris(dodecyloxy)benzoyl)oxy)phenyl)diazenyl)benzenesulfonic acid at different degrees of neutralization (DN) was addressed by small- and wide-angle neutron scattering technique. To get information on the conformation of P2VP chains spatially confined within lamellar and columnar mesophases at different DNs, the complexes were prepared from a blend of protonated and deuterated P2VP. The 2D scattering patterns of extruded fibers show that at low DN the complex self-organizes in a lamellar phase. The inertial mean distances in the direction parallel and perpendicular to the fiber axis reveal the formation of compact disc-like globules of individual $\mathrm{P} 2 \mathrm{VP}$ chains at $\mathrm{DN}=25$ and $33 \%$. With the increase of $\mathrm{DN}$ the interaction between the neighboring side groups along the backbone increases. This results in formation of hexagonal columnar phase at $\mathrm{DN}=50 \%$, in which the P2VP macromolecules are confined within cylindrical channels forming most probably a disordered helical conformation. At $\mathrm{DN}=1.0$, the enhanced rigidity of the complex hinders the accommodation of the ligands at the interface with polymer chain. This results in appearance of non-bonded side groups in the columns giving rise to a poorly-ordered structure. In contrast to more rigid chains of poly(4-vinylpyridine), the transition from lamellar to columnar mesophase in P2VP occurs at lower DNs, indicating a significant role of the chain flexibility in the formation of ordered structures.
\end{abstract}

Keywords: Chain conformation, small-angle neutron scattering, supramolecular complexes 


\section{Introduction}

Conformation is one of the most important characteristics of the polymer chain [1]. Synthetic linear macromolecules generally adopt the random coil conformation in melts and amorphous glasses, while in the semicrystalline state the chains tend to fold back and forth to form several-nanometerthick crystalline lamellae [2]. A classical technique for studies of polymer chain conformation is small-angle neutron scattering (SANS) [3]. SANS allows for contrasting of particular parts of organic molecules by substitution of hydrogen atoms by deuterium. This makes it possible to obtain information on the structure of deuterium-decorated fragments of complex systems due to a strong difference in the scattering cross-sections for hydrogen and deuterium. In contrast to dynamic light scattering and small-angle X-ray scattering, SANS allows assessing polymer conformation in the solid state. For example, detailed studies of crystallization of the blends of protonated and deuterated linear polymers showed that adjacent reentry fold model is very improbable and the radius of gyration of the chain stays almost invariable during transition from the amorphous to crystalline state $[4,5]$. In a number of reports the SANS technique was employed to address the polymer conformation in the conditions of spatial confinement. In thin films, the conformation typical of the bulk starts to modify at the film thicknesses comparable to the radius of gyration of the chain [6,7]. For deposited ultrathin polystyrene films, SANS analysis reveals formation of 2D oblate Gaussian coils [8]. Similar results were obtained for geometrical confinement of polymer chains between nanoparticles in silica/polystyrene (PS) nanocomposites [9].

Another approach to modify the chain conformation consists in adding substituents to the backbone with side groups that can self-assemble in an ordered structure. Since the side groups are covalently connected to the backbone, the chain conformation cannot be independent of their arrangement. A well-known example is comb-like side-chain liquid-crystalline (LC) polymers where the formation of mesogenic side groups in nematic or smectic mesophase leads to anisotropy of the backbone conformation [10,11]. In case of smectic structures the macromolecules form oblate Gaussian coils with repartitioning of the backbones between the neighboring mesogen layers. 

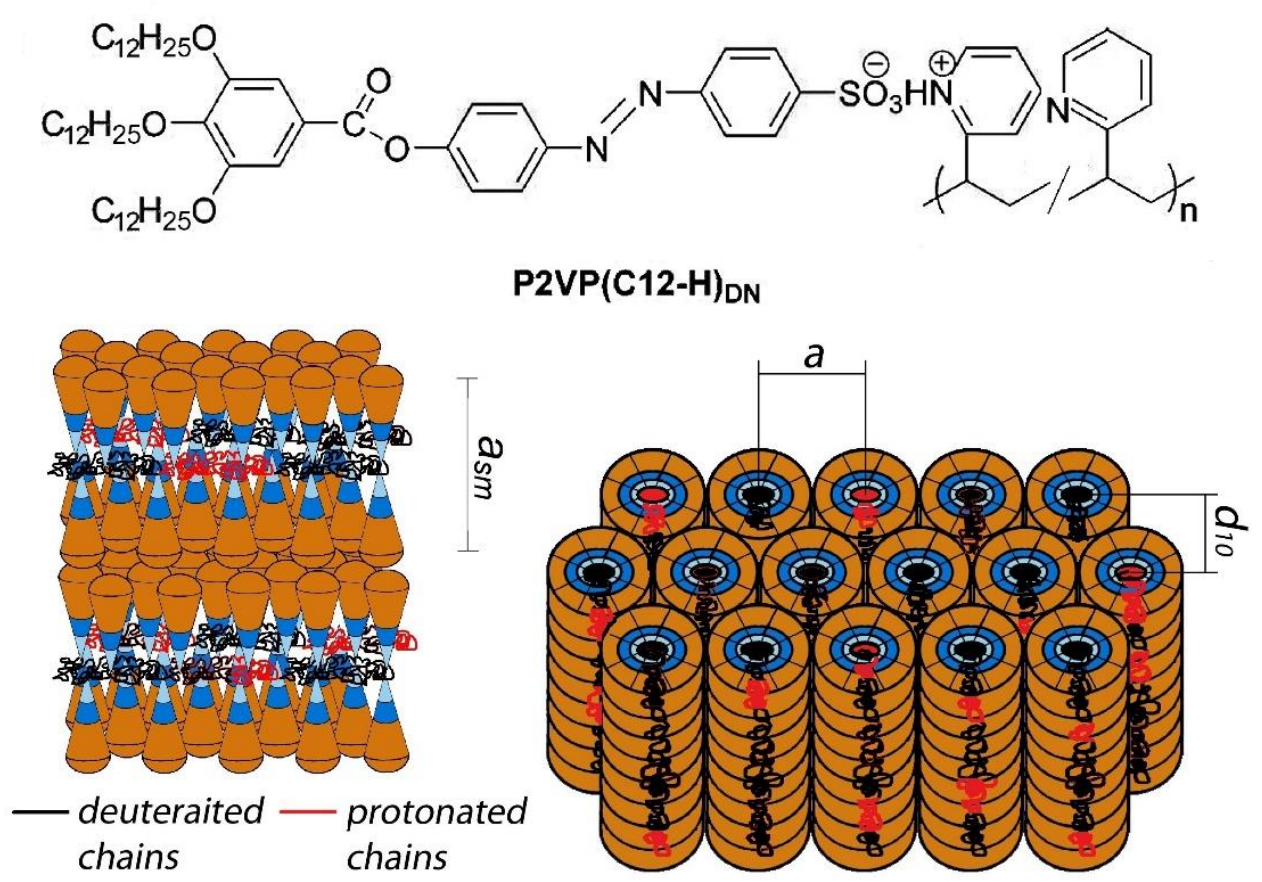

Figure 1. Chemical structure of the studied complexes with (A). Packing model of the complexes $\mathrm{P} 2 \mathrm{VP}(\mathrm{C} 12-\mathrm{H})_{\mathrm{DN}}$ in a lamellar phase $(\mathrm{DN} \leq 0.33)(\mathrm{B})$ and in hexagonal columnar phase $(\mathrm{DN} \geq$ 0.50) (C). Deuterated and protonated P2VP chains are colored in black and red, respectively.

It is well documented that macromolecules bearing wedge-shaped side groups can form columnar structures, and the self-assembly of these monodendron-side groups in a cylindrical structure can induce formation of a disordered helical conformation of the polymer backbone [12-14].

Complexation of polymer chains with low molecular weight amphiphilic molecules via noncovalent interactions such as ionic bonds and hydrogen bonding has become a standard approach for the design of nanostructured polymer materials [15-20]. In this approach, linear polymer chains and low molecular weight surfactants bind to each other to form comb-shaped supramacromolecules ("complexes"), which in turn self-assemble on a length scale of a few nanometers. Due to incompatibility between the polar polymer chain and non-polar part of the surfactant molecules, such complexes in the bulk form microphase-separated morphologies, most frequently lamellar structures. A fascinating example of a polymer complex is polyaniline protonated with camphorsulfonic acid. This complex forms a smectic phase in cast films with high degree of structural order and metallic conductivity [21,22]. The system shows a steric matching between the solvent and polymer repeating units resulting in extended chain conformation of polyaniline $[23,24]$.

Previously, we have designed a series of wedge-shaped amphiphilic molecules containing a large hydrophobic rim and a hydrophilic sulfonic acid group at the focal point, which can self-assemble into lamellar and columnar structures [25-30]. It was further demonstrated that the complexes of poly(4-vinylpyridine) (P4VP) with wedge-shaped sulfonic acid molecules exhibited a liquid- 
crystalline lamellar phase at lower degrees of neutralization (DN), while formation of a hexagonal columnar mesophase was observed at higher DN [27]. Based on small-angle X-ray scattering data, a transition of polymer chain conformation from a 2D coil in the lamellar structure to a disordered helix in the columnar phase was suggested.

In the present work, we employ small-angle (SANS) and intermediate-angle (IANS) neutron scattering technique to investigate the conformation of poly(2-vinylpyridine) (P2VP) in confined conditions of a smectic and columnar LC phases formed by complexation with 4-((4-)((3,4,5tris(dodecyloxy)benzoyl)oxy)phenyl)diazenyl)benzenesulfonic acid (cf. Fig. 1). In comparison with P4VP, the P2VP chains are more flexible, so it is expected that their conformation sheds light on the interplay between the LC structure of the ligand and polymer chain conformation.

\section{Experimental section}

The polymer complexes were prepared according to the procedure described previously [27]. A mixture of deuterated P2VP $\left(\mathrm{M}_{\mathrm{N}}=25 \mathrm{~K}, \mathrm{M}_{\mathrm{W}}=27 \mathrm{~K}\right.$, Polymer Source Inc. $)$, in which three aromatic protons were replaced by deuterium, and protonated P2VP $\left(\mathrm{M}_{\mathrm{N}}=27 \mathrm{~K}, \mathrm{M}_{\mathrm{w}}=28 \mathrm{~K}\right.$, Polymer Source Inc.) of a similar molecular weight was dissolved in chloroform and used to prepare complexes with different DN values, namely 25,33, 50 and $100 \%$.

The isotropization temperature measured by polarizing optical microscopy was found to be 277 , $276,278{ }^{\circ} \mathrm{C}$ and $265^{\circ} \mathrm{C}$ for the complexes with DN 25, 33, 50 and $100 \%$, respectively.

DSC measurements were performed according to the procedure described in our previous paper [3]. The data were recorded in the range from -50 to $250^{\circ} \mathrm{C}$ with a heating rate of $10^{\circ} \mathrm{C} / \mathrm{min}$ using Netzsch DCS 214 Polyma calorimeter. Samples (typical weight: $3 \mathrm{mg}$ ) were enclosed in standard Netzsch $25 \mu$ aluminum pans. Phase transition temperature $T_{\text {tr }}$ and enthalpy $\Delta H_{\text {tr }}$ were identified as onset and area of the endothermic peak on the second heating scans, respectively.

Intermediate-angle X-ray scattering (IAXS) measurements on isotropic samples were carried out on the BM26 beamline of the European Synchrotron Radiation Facility (Grenoble, France). IAXS images were recorded with the help of a Freelon $2 \mathrm{~K}$ detector with sample-to-detector distance of $3 \mathrm{~m}$ using wavelength of $1.24 \AA$. The modulus of the scattering vector $q=4 \pi \sin (\theta) / \lambda$, where $\theta$ is the Bragg angle and $\lambda$ the wavelength was calibrated using several orders of silver behenate.

To calculate parameters of anisotropic conformation of individual P2VP molecules in the LC phase, oriented fibers of complexes with deuterated P2VP species in the protonated matrix were prepared. The fraction of deuterated P2VP chains was varied from 10 to $100 \%$. The samples are denoted as $\mathrm{P} 2 \mathrm{VP}(\mathrm{C} 12-\mathrm{H})_{\mathrm{DN}}-\mathrm{f}$, where $\mathrm{DN}$ is the neutralization degree and $\mathrm{f}-$ the fraction of deuterated chains. The oriented fibers were extruded at $130^{\circ} \mathrm{C}$ in the liquid-crystalline state and consequently annealed at $120^{\circ} \mathrm{C}$ for $3 \mathrm{~h}$. 
The neutron scattering measurements were performed on the D22 instrument of Laue-Langevin Institute (Grenoble, France) with a 2D multi detector at $\lambda=4.5 \AA$. The sample-to-detector distances were $2 \mathrm{~m}$ for intermediate -angle (IANS) and $11 \mathrm{~m}$ for small-angle (SANS) neutron scattering. The signal from a purely protonated sample was used as background for subtraction from the patterns. The $d$-spacings of the LC phase were calculated from the modulus of the scattering vector $\mathrm{q}$ according to: $d=2 \pi / q$.

\section{Results and discussion}

Phase composition of the studied complexes was identified by combination of DSC and WAXS techniques. A single endothermic peak in the range $100-210^{\circ} \mathrm{C}$ was observed for all the studied complexes (Fig. 2). This peak can be associated with local disordering of alkyl chains without distortion of the LC phase [3]. With DN increase from 0.25 to 0.5 the transition temperature gradually increases and enthalpy decreases (Table 1). The P2VP(C12-H)1-0.1 complex shows a drastic increase of the $\mathrm{T}_{\text {tr }}$ without significant change of $\Delta \mathrm{H}_{\mathrm{tr}}$. This effect is probably related to a smaller enthalpy change during transition for this sample. The clearing point $\left(260-280^{\circ} \mathrm{C}\right)$ identified by POM cannot be detected by DSC, which can be accounted for by a very small enthalpy variation across this transition.

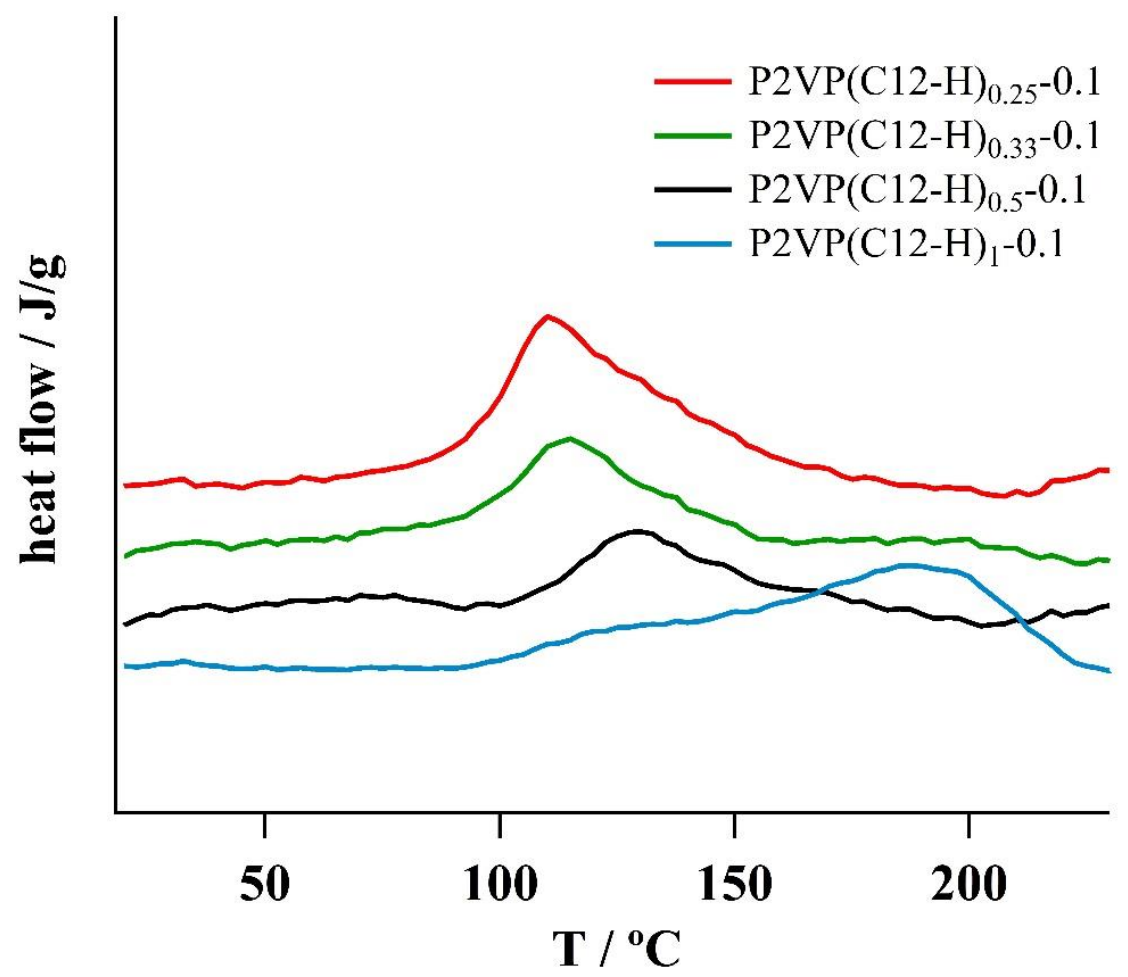

Figure 2. DSC curves corresponding to the second heating run of $\mathrm{P} 2 \mathrm{VP}(\mathrm{C} 12-\mathrm{H})_{1}-0.1$ (blue curve), $\mathrm{P} 2 \mathrm{VP}(\mathrm{C} 12-\mathrm{H})_{0.5}-0.1$ (black curve), $\mathrm{P} 2 \mathrm{VP}(\mathrm{C} 12-\mathrm{H})_{0.33}-0.1$ (green curve), and $\mathrm{P} 2 \mathrm{VP}(\mathrm{C} 12-\mathrm{H})_{0.25}-0.1$ (red curve). 
Table 1. Thermal parameters of the complexes determined by DSC

\begin{tabular}{lllll}
\hline $\mathrm{DN}, \%$ & 100 & 50 & 33 & 25 \\
$\mathrm{~T}_{\mathrm{tr}},{ }^{\circ} \mathrm{C}$ & 154 & 111 & 93 & 92 \\
$\Delta \mathrm{H}_{\mathrm{tr}}, \mathrm{J} / \mathrm{g}$ & 3.53 & 2.98 & 3.51 & 7.01 \\
\hline
\end{tabular}

The identification of the LC phase was performed by the IAXS analysis (Fig. 3). On the WAXS curves of the $\mathrm{P} 2 \mathrm{VP}(\mathrm{C} 12-\mathrm{H})_{0.25}-0.1$ and $\mathrm{P} 2 \mathrm{VP}(\mathrm{C} 12-\mathrm{H})_{0.33}-0.1$ samples one can observe two peaks with q-values ratio of 2:1, which is typical for a smectic phase. In contrast, the IAXS patterns of the P2VP(C12-H $)_{0.5}-0.1$ complex exhibit three intense reflections with q-values given by the ratio 1:sqrt(3):2 (cf. black curve in Fig. 3). These reflections were indexed as 10, 11 and 20 of a hexagonal columnar $\left(\mathrm{Col}_{h}\right)$ phase. For complex P2VP $(\mathrm{C} 12-\mathrm{H})_{1}-0.1$ the intensity of the observable peaks is much smaller but the ratio of the corresponding q-values $1: \operatorname{sqrt}(3): 2: \operatorname{sqrt}(7)$ can also be attributed to a Col $\mathrm{h}_{\mathrm{h}}$ phase (cf. blue curve in Fig. 3,).

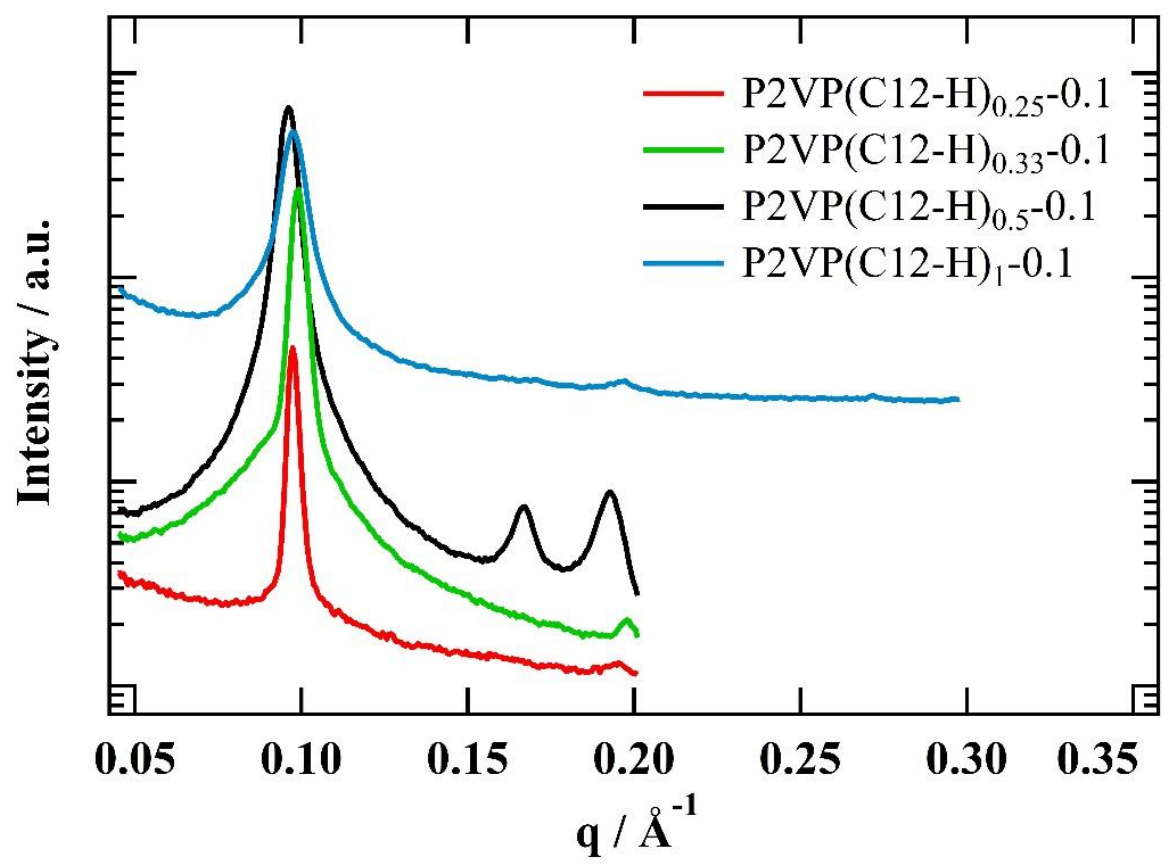

Figure 3. 1D IAXS profiles of the studied complexes with different degrees of neutralization, as indicated in the figure legend.

The analysis of oriented SANS and WANS patterns of the complexes normalized by the concentration of deuterated P2VP shows that at low concentration of the deuterated chains (5-10 wt.-\%) the intensity and shape of the integrated profiles is largely independent of their volume fraction (Fig. 4). In this case, the deuterated P2VP chains can be considered as individual objects in the protonated matrix. However, at the fraction of $50 \mathrm{wt} .-\mathrm{\%}$ the profile changes significantly due to interference between the neighboring deuterated P2VP macromolecules. For further data 
treatment, the samples with $10 \mathrm{wt} .-\%$ deuterated fraction have been selected as the most optimal ones.

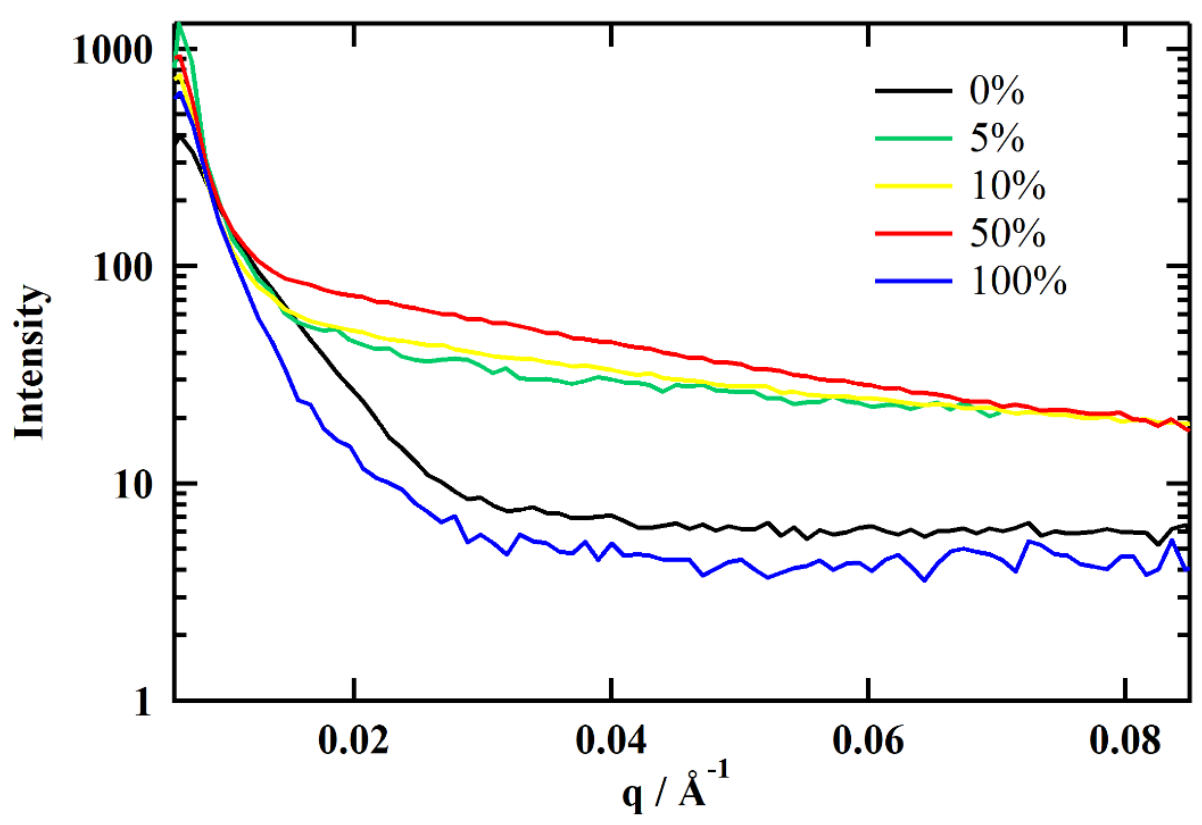

Figure 4. 1D SANS profiles of $\mathrm{P} 2 \mathrm{VP}$ as a function of the fraction of deuterated chains.

The 1D curves obtained by integration 2D IANS patterns of oriented fibers of complexes confirm the LC structures determined by IAXS (Fig. 5). For P2VP(C12-H $)_{0.25-0.1}$ and P2VP(C12-H $)_{0.33-}$ 0.1 two reflections of a smectic phase can be detected (Fig. 5, red and green curves). On the diffractogramms of the fibers with higher DNs one can see two well-pronounced peaks with qvalues ratio of 1 :sqrt(3) corresponding to a $\mathrm{Col}_{\mathrm{h}}$ phase (Fig. 5, black and blue curves). For the $\mathrm{P} 2 \mathrm{VP}(\mathrm{C} 12-\mathrm{H})_{0.5}-0.1$ fiber the 11 and 20 reflections cannot be resolved because of instrumental broadening and non-monochromatic neutron wavelength. The calculated parameters of the smectic and columnar phases are summarized in Table 2. One can see that the parameters of both $\mathrm{Col}_{\mathrm{h}}$ and smectic liquid-crystalline lattices increase with the increase of DN. Importantly, the WANS patterns show orientation of diffraction peaks' intensity in the direction normal to the fiber axis (Fig. 6B,D). Such texture corresponds to orientation of columns and smectic layers parallel to the fiber direction.

The difference in the molecular organization of the complexes is clearly determined by specific conformation of the polymer chain (Fig. 1B,C). Taking into account the geometrical size of the ligand with extended dodecyl side chains (29 $\AA$ ), one can see that in the smectic phase the polymer chain is confined in a narrow slit (of approx. $10 \AA$ ) between the layers of wedge-shaped molecules. By contrast, in the columnar phase, the P2VP chain is localized within cylindrical channels. 


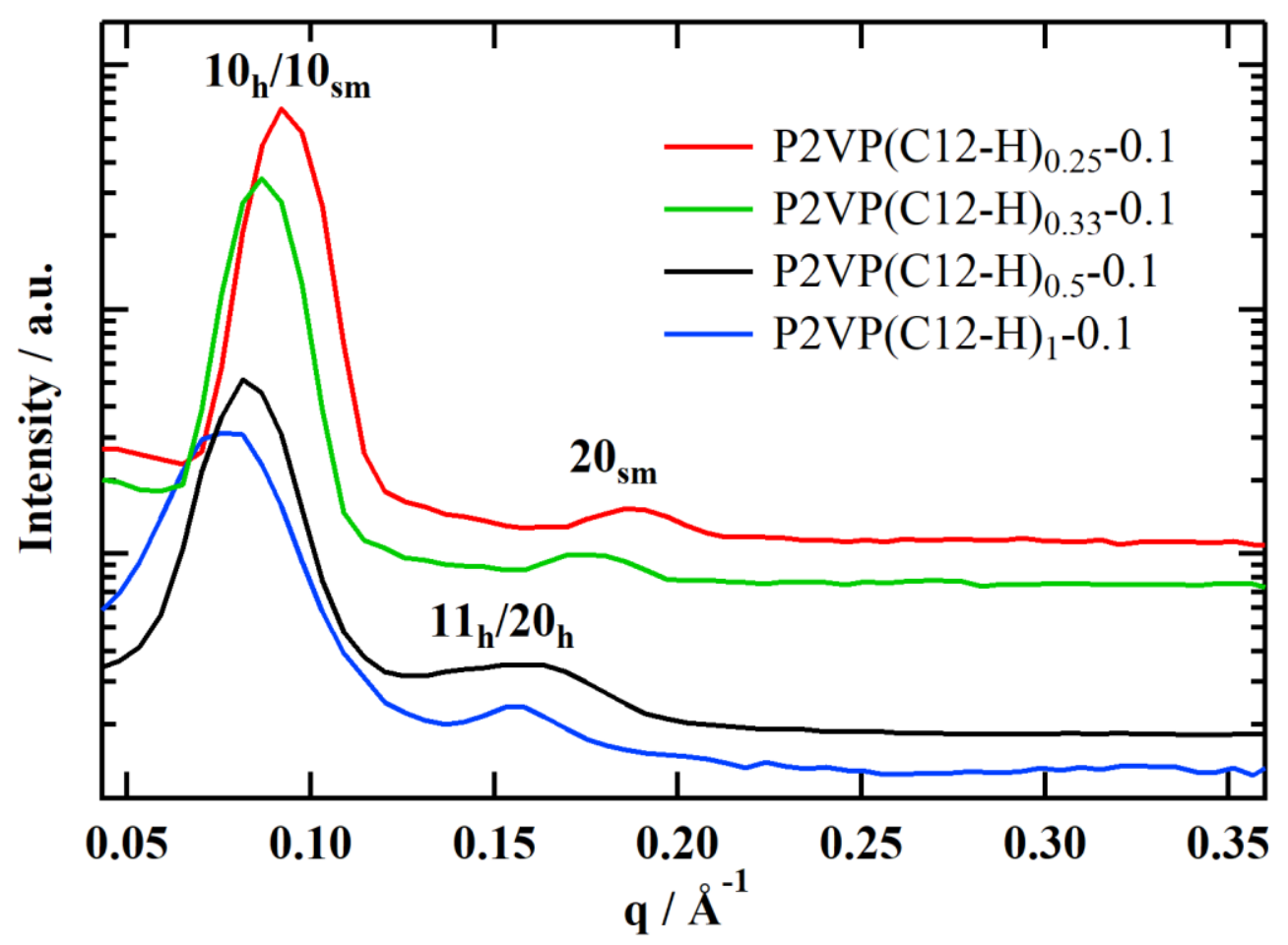

Figure 5. 1D IANS profiles of the complexes with different degrees of neutralization.

The intensity of coherent neutron scattering is given by the following formula [31] :

$I(q) \cong V^{-1}\left(a_{h}-a_{d}\right)^{2} S(q)$

where $a_{h}$ and $a_{d}$ are the scattering lengths of deuterated and protonated repeat units of volume $\mathrm{V}$, respectively, and $\mathrm{S}(\mathrm{q})$ - the structure factor. Assuming weak interaction between the monomers, the structure factor reads as follows:

$S(q)^{-1}=\left(\phi_{h} N_{h} P_{h}\left(q R_{g}\right)\right)^{-1}+\left(\phi_{d} N_{d} P_{d}\left(q R_{g}\right)\right)^{-1}-2 \chi$

where $\chi$ is the interaction parameter, $\phi_{\mathrm{h}}$ and $\phi_{\mathrm{d}}=1-\phi_{\mathrm{h}}$ are the volume fractions of the protonated chains and $\mathrm{R}_{\mathrm{g}}, \mathrm{N}_{\mathrm{h}}, \mathrm{N}_{\mathrm{d}}, \mathrm{P}_{\mathrm{h}}, \mathrm{P}_{\mathrm{d}}$ are the radii of gyration, polymerization degrees and form-factors of the two species, respectively [32,33]. For low concentrations of deuterated chains eq. (2) can be expressed in the range of small q-values ( $\left.\mathrm{qR}_{\mathrm{g}}<<1\right)$ using Zimm approximation [34]:

$S(q)^{-1} \cong A \exp \left(\left\langle x^{2} \alpha^{2}+y^{2} \beta^{2}+z^{2} \gamma\right\rangle q^{2}+\cdots\right)$

where $(\alpha, \beta, \gamma)$ are the projections of a unitary vector parallel to $\mathbf{q}$ in the basis of the main rotational axes $\mathrm{x}, \mathrm{y}$ and z. (cf. Fig. 1) For the case of fiber symmetry, the scattering intensity profiles extracted 
from 2D patterns along $\left(I_{z}\right)$ and normal $\left(I_{x y}\right)$ to the fiber direction (cf. Figure 4, left) can be written as follows:

$$
\begin{aligned}
& I_{z}(q)^{-1} \cong K \exp \left(q^{2} R_{z}^{2}+\cdots\right) \cong K\left(1+q^{2} R_{z}^{2}\right) \\
& I_{x y}(q)^{-1} \cong K \exp \left(q^{2} R_{x y}^{2}+\cdots\right) \cong K\left(1+q^{2} R_{x y}^{2}\right)
\end{aligned}
$$

where $K$ is proportional to the difference of the mean square scattering lengths and interface area, and $R_{z}$ and $R_{x y}$ are the inertial mean distances of isolated deuterated P2VP chains along and normal to the fiber direction, respectively. Calculation of the R-values for the amorphous P2VP sample with a radius of gyration $\mathrm{R}_{\mathrm{g}}: \mathrm{R}=\mathrm{R}_{\mathrm{g}} / \sqrt{3}$, shows good agreement with the parameters of the Gaussian coil measured by the light scattering technique (cf. Table 2) [25].

Table 2. Parameters of $L C$ lattice and corresponding inertial mean distances of the complexes with different $\mathrm{DN}$-values

\begin{tabular}{ccccccc}
\hline DN,\% & Phase & $\mathrm{a}, \AA$ & \multicolumn{2}{c}{$\mathrm{R}$ experimental, $\AA$} & \multicolumn{2}{c}{$\mathrm{R}$ calculated*, $\AA$} \\
\cline { 3 - 7 } & & & $\mathrm{R}_{\mathrm{xy}}$ & $\mathrm{R}_{\mathrm{z}}$ & $\mathrm{R}_{\mathrm{xy}}$ & $\mathrm{R}_{\mathrm{z}}$ \\
\hline 0 & - & 23.2 & 23.2 & 24.5 & 24.5 \\
25 & smectic & 67.7 & 15.9 & 16.1 & 12.8 & 11.4 \\
33 & smectic & 72.4 & 19.1 & 16.4 & 17.8 & 13.5 \\
50 & Col $_{\mathrm{h}}$ & 92.4 & 9.7 & 24.2 & 8.6 & 18.6 \\
100 & Col $_{\mathrm{h}}$ & 95.1 & 11.9 & 25.1 & 9.3 & 37.0 \\
\hline
\end{tabular}

*The value corresponds to conformation of the compact globule.

However, because of interaction between the bulky side groups the conformation of the chain in the smectic layer (low DN) is far from a 2D Gaussian coil $\left(R_{g}=158 \AA\right)$ [35]. We suppose that the conformation is mainly determined by the distance between the neighboring side groups along the P2VP backbone. In the case of a strong aggregation the macromolecule with the bound mesogens forms a compact object.

Let us assume that in the layer between the mesogens a P2VP molecule adopts a disc-like shape with radius $r$ and thickness $h$. The inertial mean distance of the chain in such a "compact" globule can be calculated as [10]:

$R_{\alpha}^{2}=\frac{h^{2} \cos ^{2} \alpha}{12}+\frac{r^{2} \sin ^{2} \alpha}{4}$

where $\alpha$ is the angle between the axis of the disc and scattering vector $\mathbf{q}$. Taking into account uniaxial orientation of smectic layers parallel to fiber direction, the disc axis is randomly oriented in the plane normal to the fiber axis. Consequently: 
$R_{x y}=\left\langle R^{2}\right\rangle_{\alpha}^{1 / 2}=\sqrt{\frac{r^{2}}{8}+\frac{h^{2}}{24}}=\sqrt{\frac{s_{l} * N * D N}{16 \pi}+\frac{h^{2}}{24}}$

$R_{z}=R_{\alpha=\pi / 2}=\frac{r}{2}=\sqrt{\frac{s_{l} * N * D N}{8 \pi}}$

where $s_{l}=54.6 \AA^{2}$ is the cross-section of one mesogen in the layer, $N=255$ - degree of polymerization and DN - neutralization degree. The thickness of the gap $h$ between the mesogenic layers, where P2VP chain is confined, can be calculated from the smectic d-spacing $a_{\text {sm }}$ and contour length of the mesogen $l \sim 29 \AA$ as $h=\mathrm{a}_{\mathrm{sm}}-2 l$. The calculated values are given in Table 2 .

For low DNs the experimental values of $\mathrm{R}_{\mathrm{xy}}$ and $\mathrm{R}_{\mathrm{z}}$ significantly exceed the calculated ones. Such difference indicates a weak correlation between the mesogens bonded to the same P2VP molecule leading to partial interpenetration of several macromolecules in the layer. With the increase of DN to 0.33 the average distance between the mesogens on the chain decreases. In the result, during formation of a LC phase the side groups prefer to pack with mesogens pertinent to the same chain, which adopts a conformation close to that of a compact quasi-2D globule.

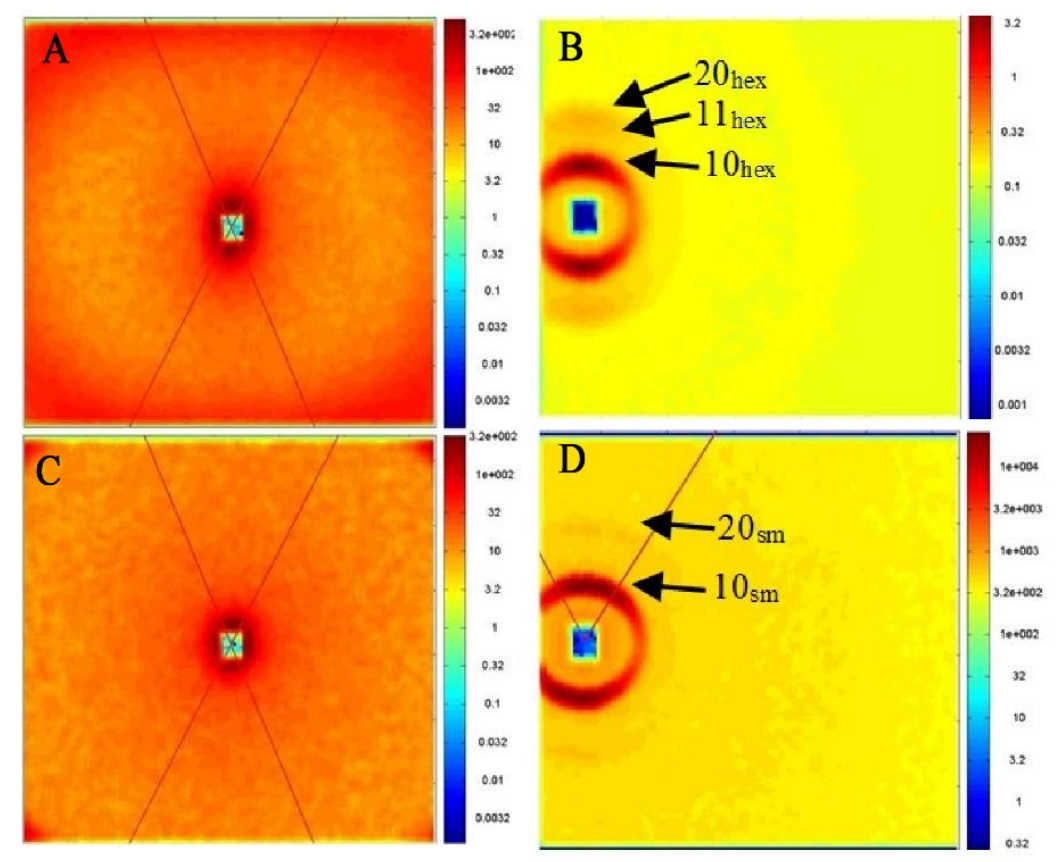

Figure 6. 2D SANS $(A, C)$ and IANS $(B, D)$ patterns of complexes $\mathrm{P} 2 \mathrm{VP}(\mathrm{C} 12-\mathrm{H})_{0.5}-0.1(\mathrm{~A}, \mathrm{~B})$ and $\mathrm{P} 2 \mathrm{VP}(\mathrm{C} 12-\mathrm{H})_{0.25}-0.1$ (C,D). The fiber direction is horizontal. Z-scale is logarithmic. Red lines show angular sectors of integration in the vertical direction.

Further increase of DN in the smectic phase is limited by the density of binding centers at the interface between the polymer and ligands. Because of the wedge-shaped geometry of the mesogens this density can be increased by self-assembling in columns (Fig.1B). The area per ligand in the polymer-side groups interface $s_{c}$ and inner radius of the interface $r_{i}$ can be estimated from the parameters of the hexagonal lattice and macroscopic density of the samples $(\rho=1.18$ $\left.\mathrm{g} / \mathrm{cm}^{3}\right)$. The found values of $s_{c}\left(s_{c}=18.7\right.$ and $17.2 \AA^{2}$ for $\mathrm{DN}=1$ and 0.5 , respectively) are 
significantly smaller than the mesogen cross-section in the layer $\left(54.6 \AA^{2}\right)$. Similarly to the smectic structure, the chain conformation in the case of compact globule corresponds to a cylinder of radius $r_{i}$ and height $H$ with the main axis parallel to the fiber direction. The $\mathrm{R}_{\mathrm{xy}}$ and $\mathrm{R}_{\mathrm{z}}$ are calculated as following (Table 2):

$R_{z}=\frac{H}{\sqrt{12}}=\frac{s_{c} * N * D N}{4 \sqrt{3} \pi r_{i}} ; \quad R_{x y}=\frac{r_{i}}{2}$

As one can see from Table 2, in the direction normal to the columns the experimental and calculated values of $\mathrm{R}_{\mathrm{xy}}$ show a good agreement for the samples $\mathrm{P} 2 \mathrm{VP}(\mathrm{C} 12-\mathrm{H})_{0.5}-0.1$ and $\mathrm{P} 2 \mathrm{VP}(\mathrm{C} 12-\mathrm{H})_{1}-0.1$. In the direction along the column the experimental value of $\mathrm{R}_{\mathrm{z}}$ for sample $\mathrm{P} 2 \mathrm{VP}(\mathrm{C} 12-\mathrm{H})_{0.5}-0.1$ is higher than the one calculated from eq.7. The found parameters suggest the formation of a slightly extended conformation of the polymer chain in the cylindrical channel for $\mathrm{DN}=50 \%$, which is most probably a disordered helical conformation as proposed in our previous publication [27]. At higher DNs the $\mathrm{R}_{\mathrm{z}}$ of the P2VP chain in the compact globule (which is the densest conformation) exceeds the experimental one. One can suppose that at $\mathrm{DN}=100 \%$ the helical conformation of the polymer chain in cylindrical confinement cannot provide the required density of the pyridinium groups at the interface, leading to appearance of non-bonded mesogens in the columns and disturbance of the hexagonal structure. In this case, the ligand molecules also lack freedom to diffuse along the polymer chains, which could be a mechanism for them to improve order [36] The formation of such disturbed columns can explain the low intensity of 11 and 20 peaks recorded for sample $\mathrm{P} 2 \mathrm{VP}(\mathrm{C} 12-\mathrm{H})_{1}-0.1$. It is interesting to note that superior density of the linking centers can be obtained in spherical globules organized on a cubic lattice. However, the formation of a cubic phase was not observed here probably due to a specific planar shape of the ligands or inappropriate thermal processing.

\section{Conclusions}

Using small- and wide-angle neutron scattering the liquid-crystalline structure and chain conformation in the complexes of the P2VP chains with wedge-shaped amphiphilic sulfonic acid ligands were studied. The chain conformation of oriented LC fibers was quantitatively described in terms of inertial mean distances in two directions: parallel and perpendicular to the fiber axis. It was shown that at low DN, the complexes self-organize in a smectic phase with conformation of the chain close to that of a disc. With the increase of DN the interaction between neighboring side groups increases resulting in compactization of the chain in the layer. At $\mathrm{DN}>33 \%$ the density of the binding centers in the layer was found to be insufficient to accommodate the polymer chain resulting in formation of a columnar hexagonal phase at $\mathrm{DN}=50 \%$. As compared to $\mathrm{P} 4 \mathrm{VP}$, this lamellar-to-columnar transition occurs in the case of the more flexible P2VP chains at lower DNs. 
At $\mathrm{DN}=100 \%$, the rigidity of the complex does not provide anymore the required density of the linking centers to complex all the ligand molecules on the cylindrical polymer/ligand interphase, thereby leading to formation of poorly-ordered irregular columns. The obtained results emphasize the role of the chain conformation in self-assembly of the polymer-mesogen complexes and their thermodynamic stability.

Acknowledgment

The research was supported by the Russian Science Foundation (project No 16-13-10369).

References

[1] J.P. Cotton, B. Farnoux, G. Jannink, C. Picot, G.C. Summerfield, Polymer configuration in bulk: neutron diffraction by deuterated polymers in a nondeuterated matrix, J Polym Sci, Part C, Polym Symp 42 (2017) 807-815, https://doi.org/10.1002/polc.5070420230.

[2] B. Wunderlich, Macromolecular physics. Vol.1, Crystal structure, morphology, defects (1973), https://doi.org/10.1016/B978-0-12-765601-4.X5001-X.

[3] R.J. Roe, Methods of x-ray and neutron scattering in polymer science, Oxford University Press, Oxford, 2000.

[4] M. Stamm, E.W. Fischer, M. Dettenmaier, P. Convert, Chain conformation in the crystalline state by means of neutron scattering methods, Faraday Discuss. Chem. Soc. 68 (1979) 263-278, https://doi.org/10.1039/DC9796800263.

[5] M. Stamm, Influence of the Conformation of Polyethylene on Wide-Angle Neutron Scattering Patterns, 20 (1982) 235-244, https://doi.org/10.1002/pol.1982.180200206.

[6] A. Brûlet, F. Boué, A. Menelle, J.P. Cotton, Conformation of polystyrene chain in ultrathin films obtained by spin coating, Macromolecules. 33 (2000) 997-1001, https://doi.org/10.1021/ma9906783.

[7] Z. Xu, N. Hadjichristidis, L.J. Fetters, J.W. Mays, Structure/chain-flexibility relationships of polymers, 120 (1995) 1-50, https://doi.org/10.1109/CCTAE.2010.5543623.

[8] R.L. Jones, S.K. Kumar, D.L. Ho, R.M. Briber, T.P. Russell, Chain conformation in ultrathin polymer films, Nature. 400 (1999) 146-149, https://doi.org/10.1038/22080.

[9] N. Jouault, F. Dalmas, S. Said, E. Di Cola, R. Schweins, J. Jestin, F. Boué, Direct measurement of polymer chain conformation in well-Controlled model nanocomposites by combining SANS and SAXS, Macromolecules. 43 (2010) 9881-9891, https://doi.org/10.1021/ma101682t.

[10] J.P. Cotton, F. Hardouin, Chain Conformation of Liquid-Crystalline Polymers Studied By Small-Angle Neutron, Prog.Polym.Sci. 22 (1997) 796-826, https://doi.org/10.1016/S0079-6700(97)00007-5. 
[11] L. Noirez, P. Keller, J.P. Cotton, On the structure and the chain conformation of sidechain liquid crystal polymers, Liq. Cryst. 18 (1995) 129-148,

https://doi.org/10.1080/02678299508036602.

[12] H. Lu, X. Zeng, G. Ungar, C. Dressel, C. Tschierske, The Solution of the Puzzle of Smectic-Q: The Phase Structure and the Origin of Spontaneous Chirality, Angewandte Chemie International Edition Volume 57 (2018) 2835-2840, https://doi.org/10.1002/anie.201712812

[13] V. Percec, C.-H. Ahn, G. Ungar, D.J.P. Yeardley, M. Möller, S.S. Sheiko, Controlling polymer shape through the self-assembly of dendritic side-groups, Nature. 391 (1998) 161-164, https://doi.org/10.1038/34384.

[14] Y. Zhu, M. Zheng, Y. Tu, X.-F. Chen, Supramolecular Fluorescent Polymers Containing $\alpha$-Cyanostilbene-Based Stereoisomers: Z/E-Isomerization Induced Multiple Reversible Switching, Macromolecules 51 (2018) 3487-3496, https://doi.org/10.1021/acs.macromol.8b00347.

[15] S.-J. Wang, Y.-S. Xu, S. Yang, E.-Q. Chen, Phase behavior of a hydrogen-bonded polymer with lamella-to-cylinder transition: Complex of poly(4-vinylpyridine) and small dendritic benzoic acid derivative, Macromolecules 45 (2012) 8760-8769, https://doi.org/10.1021/ma301783h.

[16] L. Li, M. Rosenthal, H. Zhang, J.J. Hernandez, M. Drechsler, K.H. Phan, S. Rütten, X. Zhu, D.A. Ivanov, M. Möller, Light-switchable vesicles from liquid-crystalline homopolymer-surfactant complexes, Angewandte Chemie - International Edition 51 (2012) 11616-11619, https://doi.org/10.1002/anie.201205660.

[17] Z. Chen, R. Liu, B. Ren, Ionically self-assembled thermotropic liquid crystalline complexes of linear polysiloxane and dendritic amphiphiles, Journal of Macromolecular Science, Part B: Physics 51 (2012) 1172-1185, https://doi.org/10.1080/00222348.2011.625908.

[18] G.A. Ferreira, W. Loh, Liquid crystalline nanoparticles formed by oppositely charged surfactant-polyelectrolyte complexes, Current Opinion in Colloid and Interface Science 32 (2017) 11-22, https://doi.org/10.1016/j.cocis.2017.08.003.

[19] J.J. Hernandez, H. Zhang, Y. Chen, M. Rosenthal, M.D. Lingwood, M. Goswami, X. Zhu, M. Moeller, L.D. Madsen, D.A. Ivanov, Bottom-Up Fabrication of Nanostructured Bicontinuous and Hexagonal Ion-Conducting Polymer Membranes, Macromolecules 14 (2017) 5392-5401, https://doi.org/10.1021/acs.macromol.6b02674.

[20] W.H. De Jeu, T. Markkula, K. Albrecht, D. A. Ivanov, M. Möller, A. Mourran, Complexing P2VP and P2VP-b-PEO with Wedge-Shaped Amphiphiles, Macromolecules 50 (2017) 4754-4758, https://doi.org/10.1021/acs.macromol.7b00720. 
[21] T. Kozik, M. Śniechowski, W. Łużny, A. Proń, D. Djurado, Neutron diffraction study of conducting polyaniline doped with $( \pm$ ) camphorsulfonic acid, Polymer 111 (2017) 148155, https://doi.org/10.1016/j.polymer.2017.01.034.

[22] O.Y. Posudievsky, O.A. Kozarenko, I.E. Kotenko, O.P. Boiko, A.G. Shkavro, V.G. Koshechko, V.D. Pokhodenko, Metallic Conductivity of Mechanochemically Doped Polyaniline, Theoretical and Experimental Chemistry 50 (2014) 197-203, https://doi.org/10.1007/s11237-014-9373-7.

[23] K. Krukiewicz, A. Katunin, The effect of reaction medium on the conductivity and morphology of polyaniline doped with camphorsulfonic acid, Synthetic Metals 214 (2016) 45-49, https://doi.org/10.1016/j.synthmet.2016.01.017.

[24] O. Abdulrazzaq, S.E. Bourdo, V, Saini, F. Watanabe, B. Barnes, A. Ghosh, A.S. Biris, Tuning the work function of polyaniline via camphorsulfonic acid: An X-ray photoelectron spectroscopy investigation, RSC Advances 5 (2015) 33-40, https://doi.org/10.1039/c4ra11832d.

[25] X. Zhu, B. Tartsch, U. Beginn, M. Möller, Wedge-Shaped Molecules with a Sulfonate Group at the Tip_A New Class of Self-Assembling Amphiphiles, Chem. - A Eur. J. 10 (2004) 3871-3878, https://doi.org/10.1002/chem.200400050.

[26] X. Zhu, M.A. Scherbina, A. V. Bakirov, B. Gorzolnik, S.N. Chvalun, U. Beginn, M. Möller, Methacrylated Self-Organizing 2,3,4-Tris(alkoxy)benzenesulfonate: A New Concept Toward Ion-Selective Membranes, Chem. Mater. 18 (2006) 4667-4673, https://doi.org/10.1021/cm060305y.

[27] X. Zhu, U. Beginn, M. Möller, R.I. Gearba, D. V. Anokhin, D.A. Ivanov, Selforganization of polybases neutralized with mesogenic wedge-shaped sulfonic acid molecules: An approach toward supramolecular cylinders, J. Am. Chem. Soc. 128 (2006) 16928-16937, https://doi.org/10.1021/ja065968v.

[28] A. Dolgopolov, K.N. Grafskaia, D. V. Anokhin, D.E. Demco, X. Zhu, D.A. Ivanov, M. Möller, Humidity-induced formation of water channels in supramolecular assemblies of wedge-shaped amphiphiles: The effect of the molecular architecture on the channel topology, Phys. Chem. Chem. Phys. 19 (2017) 7714-7720, https://doi.org/10.1039/c6cp08087a.

[29] K.N. Grafskaia, J.J.H. Rueda, X. Zhu, V.M. Nekipelov, D. V. Anokhin, M. Moeller, D.A. Ivanov, Designing the topology of ion nano-channels in the mesophases of amphiphilic wedge-shaped molecules, Phys. Chem. Chem. Phys. 17 (2015) 30240-30247, https://doi.org/10.1039/c5cp05618g.

[30] K.N. Grafskaia, D. V. Anokhin, B.I. Zimka, I.A. Izdelieva, X. Zhu, D.A. Ivanov, An “on- 
off" switchable cubic phase with exceptional thermal stability and water sorption capacity, Chem. Commun. 53 (2017) 13217-13220, https://doi.org/10.1039/c7cc08003d.

[31] R.G. Alamo, J.D. Londono, L. Mandelkern, F.C. Stehling, G.D. Wignall, Phase Behavior of Blends of Linear and Branched Polyethylenes in the Molten and Solid States by SmallAngle Neutron Scattering, Macromolecules. 27 (1994) 411-417, https://doi.org/10.1021/ma00080a014.

[32] G. Hadziioannou, R.S. Stein, Neutron scattering studies of dimensions and of interactions between components in polystyrene/poly(vinyl methyl ether) and poly(vinylidene fluoride)/poly(methyl methacrylate) amorphous blends, Macromolecules. 17 (1984) 567 573, https://doi.org/10.1021/ma00134a010.

[33] M. Warner, J. Higgins, A.J. Carter, Chain dimensions and interaction parameters in neutron scattering from polymer blends with a labeled component, Macromolecules. 16 (1983) 1931-1935, https://doi.org/10.1021/ma00246a024.

[34] P.-G. Gennes, Scaling Concepts in Polymer Physics, Cornell University Press, 1979.

[35] M. Rubinstein, L.H. Colby, Polymer Physics, Oxford University Press, London, 2003. 